T. Ono

Nagoya Math. J.

Vol. 56 (1974) 201-207

\title{
ON THE HOPF FIBRATION OVER $Z$
}

\author{
TAKASHI ONO
}

\section{§ 1. Statement of the result}

Let $h: \boldsymbol{R}^{4} \rightarrow \boldsymbol{R}^{3}$ be a quadratic map defined by

$$
h(x)=\left(x_{1}^{2}+x_{2}^{2}-x_{3}^{2}-x_{4}^{2}, 2\left(x_{2} x_{3}-x_{1} x_{4}\right), 2\left(x_{1} x_{3}+x_{2} x_{4}\right)\right) .
$$

For a natural number $t$, put

$$
\begin{aligned}
& S^{3}(t)=\left\{x \in \boldsymbol{R}^{4}, x_{1}^{2}+x_{2}^{2}+x_{3}^{2}+x_{4}^{2}=t\right\}, \\
& S^{2}(t)=\left\{y \in \boldsymbol{R}^{3}, y_{1}^{2}+y_{2}^{2}+y_{3}^{2}=t\right\} .
\end{aligned}
$$

Then $h$ induces a map

$$
h_{t}: S^{3}(t) \rightarrow S^{2}\left(t^{2}\right) .
$$

Since everything is defined over $Z, h_{t}$ induces the map

$$
h_{t, z}: S^{3}(t)_{Z} \rightarrow S^{2}\left(t^{2}\right)_{Z} \text {. }
$$

Because of the presence of 2 in the last two coordinates of $h(x), h_{t, z}$ is actually a map

$$
h_{t, Z}: S^{3}(t)_{Z} \rightarrow S^{2}\left(t^{2}\right)_{Z}^{\text {even }}
$$

where

$$
S^{2}\left(t^{2}\right)_{Z}^{\text {even }}=\left\{y \in S^{2}\left(t^{2}\right)_{Z}, y_{2}, y_{3} \text { are even }\right\} .
$$

To each $y \in S^{2}\left(t^{2}\right)_{Z}^{\text {even }}$ we shall associate two numbers as follows. First, we denote by $a(y)$ the number of $x \in S^{3}(t)_{z}$ such that $h_{t, z}(x)=y$. Next, we denote by $\Delta_{y}$ the greatest common divisor of the four integers $\frac{1}{2}\left(t+y_{1}\right), \frac{1}{2}\left(t-y_{1}\right), \frac{1}{2} y_{2}, \frac{1}{2} y_{3}$. On the other hand, for a natural number $n$, denote by $r(n)$ the number of integral solutions $(X, Y)$ of the equation $X^{2}+Y^{2}=n$. It is well known that

$$
r(n)=4\left(d_{1}(n)-d_{3}(n)\right)
$$

Received June 11, 1974. 
where $d_{1}(n)$ and $d_{3}(n)$ are the numbers of divisors of $n$ of the form $4 m+1$ and $4 m+3$ respectively.

The purpose of the present paper is to prove the relation:

$$
a(y)=r\left(\Delta_{y}\right), \quad y \in S^{2}\left(t^{2}\right)_{Z}^{\text {even }}
$$

As the readers notice, (1.1) reflects the fact that each fibre of $h_{t}$ is a circle.

\section{§ 2. Change of the fibration}

Let $\boldsymbol{H}$ be the classical quaternion algebra over $\boldsymbol{R}$ with the quaternion units $1, i, j, k$, with the relations $i^{2}=j^{2}=-1, k=i j=-j i$. We shall make the following natural identifications:

$$
\begin{gathered}
C=R+R i=R^{2}, \quad H=C+C j=C^{2}=R^{4}, \\
Z[i]=Z+Z i=Z^{2}, \quad H_{Z}=Z[i]+Z[i] j=Z[i]^{2}=Z^{4} .
\end{gathered}
$$

As usual, for each $z=x+y j \in \boldsymbol{H}, x, y \in C$, we write its conjugate, trace and norm by $\bar{z}=\bar{x}-y j, \operatorname{Tr} z=\bar{z}+z$ and $N z=\bar{z} z$, respectively. In working with $\boldsymbol{H}$, we shall mean by $\boldsymbol{R}^{3}$ the subspace $\boldsymbol{R} \boldsymbol{i}+\boldsymbol{R} \boldsymbol{j}+\boldsymbol{R} \boldsymbol{k}$ $=\boldsymbol{R} i+\boldsymbol{C} j$. This space is known as the space of pure quaternions and is characterized as the set of all $z \in \boldsymbol{H}$ such that $\operatorname{Tr} z=0$.

For $z \in \boldsymbol{H}$, put

$$
h(z)=\bar{z} i z
$$

Since $\operatorname{Tr}(h(z))=0, h$ is a $\operatorname{map}: \boldsymbol{R}^{4} \rightarrow \boldsymbol{R}^{3}$. A simple calculation shows that

$$
\begin{aligned}
h(z) & =(N x-N y) i+2 \bar{x} y k \\
& =\left(x_{0}^{2}+x_{1}^{2}-y_{0}^{2}-y_{1}^{2}\right) i+2\left(x_{1} y_{0}-x_{0} y_{1}\right) j+2\left(x_{0} y_{0}+x_{1} y_{1}\right) k,
\end{aligned}
$$

where $z=x+y j, x=x_{0}+x_{1} i, y=y_{0}+y_{1} i, x_{0}, x_{1}, y_{0}, y_{1} \in R$. Hence the map (2.1) coincides with the map $h$ introduced in $\S 1$.

For $t>0$, put

$$
S^{3}(t)=\left\{z \in R^{4}, N z=t\right\}, \quad S^{2}(t)=\left\{w \in R^{3}, N w=t\right\} .
$$

Since $N(h(z))=(N z)^{2}$ by $(2.1), h$ induces a map

$$
h_{t}: S^{3}(t) \rightarrow S^{2}\left(t^{2}\right) \text {. }
$$

When $t$ is a natural number, put 


$$
S^{3}(t)_{Z}=S^{3}(t) \cap Z^{4}, \quad S^{2}(t)_{Z}=S^{2}(t) \cap Z^{3} .
$$

Then, $h_{t}$ induces a map

$$
h_{t, Z}: S^{3}(t)_{Z} \rightarrow S^{2}\left(t^{2}\right)_{Z} .
$$

Our problem is to determine the image and the fibres of the map $h_{t, \boldsymbol{z}}$. To do this, it is convenient to replace the map $h_{t}$ by a map $f_{t}$ in the following way. Namely, put

$$
\sum(t)=\left\{\sigma=(\alpha, \beta, \gamma), \alpha, \beta \in \boldsymbol{R}, \gamma \in \boldsymbol{C}, \alpha+\beta=t, N_{\gamma}=\alpha \beta\right\},
$$

and $f_{t}(z)=(N x, N y, i \bar{x} y)$ for $z=x+y j \in S^{3}(t)$.

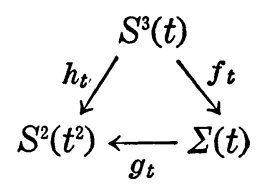

Since $N x+N y=N z=t$ and $N(i \bar{x} y)=(N x)(N y), f_{t}$ is a map $S^{3}(t) \rightarrow \Sigma(t)$. Next, put

$$
g_{t}(\sigma)=(\alpha-\beta) i+2 \gamma j, \quad \text { for } \sigma=(\alpha, \beta, \gamma) \in \Sigma(t) .
$$

Since $N\left(g_{t}(\sigma)\right)=(\alpha-\beta)^{2}+N(2 \gamma)=(\alpha-\beta)^{2}+4 \alpha \beta=(\alpha+\beta)^{2}=t^{2}, g_{t}$ is a map $\Sigma(t) \rightarrow S^{2}\left(t^{2}\right)$. If $g_{t}(\sigma)=g_{t}\left(\sigma^{\prime}\right)$ with $\sigma^{\prime}=\left(\alpha^{\prime}, \beta^{\prime}, \gamma^{\prime}\right)$, then $\alpha-\beta=\alpha^{\prime}$ $-\beta^{\prime}$ and $\gamma=\gamma^{\prime}$. Since $\alpha+\beta=\alpha^{\prime}+\beta^{\prime}=t$, we see that $g_{t}$ is injective. For any $w=u i+v j \in S^{2}\left(t^{2}\right)$, we have $w=g_{t}(\sigma)$ with

$$
\sigma=\left(\frac{1}{2}(t+u), \frac{1}{2}(t-u), \frac{1}{2} v\right) .
$$

Hence $g_{t}$ is surjective, and so bijective. Finally, it follows from (2.2) that $g_{t}\left(f_{t}(z)\right)=g_{t}(N x, N y, i \bar{x} y)=(N x-N y) i+2 i \bar{x} y j=(N x-N y) i+2 \bar{x} y k$ $=h_{t}(z)$, the commutativity of the diagram.

Now, for a natural number $t$, put

$$
\Sigma(t)_{Z}=\Sigma(t) \cap\left(Z^{2}+Z[i]\right) .
$$

Then, $f_{t}, g_{t}$ induce maps

$$
f_{t, \boldsymbol{Z}}: S^{3}(t)_{Z} \rightarrow \Sigma(t)_{\boldsymbol{Z}}, \quad g_{t, \boldsymbol{Z}}: \Sigma(t)_{\boldsymbol{Z}} \rightarrow S^{2}\left(t^{2}\right)_{\boldsymbol{Z}},
$$

respectively such that $g_{t, Z} f_{t, Z}=h_{t, Z}$. If $w=u i+v j \in S^{2}\left(t^{2}\right)_{Z}$ is in the image of $g_{t, Z}, v$ must be a multiple of 2 in $Z[i]$ and, since $N w=u^{2}+N v$ 
$=t^{2}$, both $t+u$ and $t-u$ must be even. In view of (2.3), we see that $g_{t, Z}$ is a bijection between $\Sigma(t)_{Z}$ and the set $S^{2}\left(t^{2}\right)_{Z}^{\text {eren }}=\{w=u i+v j$ $\left.\in S^{2}\left(t^{2}\right)_{Z}, 2 \mid v\right\}$. Hence, to study the map $h_{t, Z}$ is equivalent to study the $\operatorname{map} f_{t, \mathbf{Z}}$.

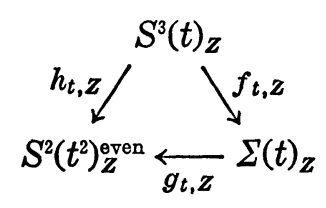

\section{§3. Existence of solutions}

Notation being as in $\S 2$, we shall determine for what $\sigma \in \Sigma(t)_{Z}$ the equation $f_{t, z}(z)=\sigma$ has a solution $z \in S^{3}(t)_{z}$. In the following, we shall put $\sigma=(\alpha, \beta, \gamma), \alpha, \beta \in \boldsymbol{Z}, \gamma=\gamma_{0}+\gamma_{1} i \in \boldsymbol{Z}[i], \gamma_{0}, \gamma_{1} \in \boldsymbol{Z}$. We shall first examine some special cases.

Case 1. $r=0$.

In this case, the relations $\alpha+\beta=t$ and $0=N \gamma=\alpha \beta$ imply that either $\alpha=0, \beta=t$ or $\alpha=t, \beta=0$, i.e. $\sigma=(0, t, 0)$ or $(t, 0,0)$. Hence $z=x+y j$ is a solution of $f_{t, z}(z)=\sigma$ if and only if either $z=y j, N y=t$ or $z=x, N x=t$. Therefore it follows that

$$
f_{t, Z}^{-1}(\sigma) \neq \emptyset \Leftrightarrow t \in N(Z[i]) .
$$

Case 2. $\gamma \neq 0$ and $\left(\gamma_{0}, \gamma_{1}\right)=1$.

Assumptions imply that $\alpha, \beta \geqq 1$. Since $\alpha \beta=N \gamma=\gamma_{0}^{2}+\gamma_{1}^{2}$, we have $\left(\gamma_{1}, \alpha\right)=1$. Therefore, there are two integers $r, s$ such that $\gamma_{0}=r \gamma_{1}+s \alpha$. Put $I=\boldsymbol{Z} \alpha+\boldsymbol{Z}(r+i)$. We claim that $I$ is an ideal. It is enough to show that $i \alpha, i(r+i) \in I$. Firstly, $i \alpha=-r \alpha+(r+i) \alpha \in I$. Secondly, we have

$$
\alpha \beta=N \gamma=\gamma_{0}^{2}+\gamma_{1}^{2}=\left(r \gamma_{1}+s \alpha\right)^{2}+\gamma_{1}^{2}=\left(1+r^{2}\right) \gamma_{1}+2 r s \gamma_{1} \alpha+s^{2} \alpha^{2},
$$

and so $\left(1+r^{2}\right) \gamma_{1}=\alpha\left(\beta-2 r s \gamma_{1}-s^{2} \alpha\right)$. Since $\left(\gamma_{1}, \alpha\right)=1, \alpha$ must divide $1+r^{2}$ : write $1+r^{2}=\alpha \alpha^{\prime}$. Then, we have

$$
i(r+i)=i r-1=r(r+i)-\alpha \alpha^{\prime} \in I,
$$

which shows that $I$ is an ideal. Since $Z[i]$ is a principal ideal ring, there is an $x \in Z[i]$ such that $I=(\bar{x})$. Hence $N x=N \bar{x}=N I=\alpha$. - Since $\gamma=\gamma_{0}+\gamma_{1} i=\left(r \gamma_{1}+s \alpha\right)+\gamma_{1} i=(r+i) \gamma_{1}+s i \alpha \in I$, we can find $y \in Z[i]$ such that $\gamma=i \bar{x} y$. Then the relation $N \gamma=\alpha \beta$ implies that $N y=\beta$. If 
we put $z=x+y j$, then we have $f_{t, z}(z)=(N x, N y, i \bar{x} y)=(\alpha, \beta, \gamma)=\sigma$. Hence $f_{t, Z}^{-1}(\sigma) \neq \emptyset$ in this case.

Case 3. $\gamma \neq 0$ and $\left(\alpha, \beta, \gamma_{0}, \gamma_{1}\right)=1$.

Put $\left(\gamma_{0}, \gamma_{1}\right)=d_{0},\left(d_{0}, \alpha\right)=d_{1}$. Hence we have $\gamma_{0}=d_{0} \gamma_{0}^{\prime}, \gamma_{1}=d_{0} \gamma_{1}^{\prime}$ with $\left(\gamma_{0}^{\prime}, \gamma_{1}^{\prime}\right)=1$ and $d_{0}=d_{1} d_{0}^{*}, \alpha=d_{1} \alpha^{*}$ with $\left(\alpha^{*}, d_{0}^{*}\right)=1$. From

$$
d_{1} \alpha^{*} \beta=\alpha \beta=N \gamma=\gamma_{0}^{2}+\gamma_{1}^{2}=d_{1}^{2} d_{0}^{* 2}\left(\gamma_{0}^{\prime 2}+\gamma_{1}^{\prime 2}\right)
$$

we get

$$
\alpha^{*} \beta=d_{1} d_{0}^{* 2}\left(\gamma_{0}^{\prime 2}+\gamma_{1}^{\prime 2}\right) \text {. }
$$

Since $d_{1}$ divides $\alpha, \gamma_{0}, \gamma_{1}$ and $\left(\alpha, \beta, \gamma_{0}, \gamma_{1}\right)=1$, we have $\left(d_{1}, \beta\right)=1$ and hence $d_{1}$ divides $\alpha^{*}: \alpha^{*}=d_{1} \alpha^{\prime}$. On the other hand, since $\left(\alpha^{*}, d_{0}^{*}\right)=1, d_{0}^{* 2}$ divides $\beta: \beta=d_{0}^{* 2} \beta^{\prime}$. Then (3.2) implies that

$$
\alpha^{\prime} \beta^{\prime}=N \gamma^{\prime}, \quad \gamma^{\prime}=\gamma_{0}^{\prime}+\gamma_{1}^{\prime} i, \quad\left(\gamma_{0}^{\prime}, \gamma_{1}^{\prime}\right)=1 .
$$

Hence, by the argument in Case 2 one can find $x^{\prime}, y^{\prime} \in Z[i]$ such that $N x^{\prime}=\alpha^{\prime}, N y^{\prime}=\beta^{\prime}, \gamma^{\prime}=i \bar{x}^{\prime} y^{\prime}$. Put $x=d_{1} x^{\prime}, y=d_{0}^{*} y^{\prime}$. Then, we have $N x=d_{1}^{2} N x^{\prime}=d_{1}^{2} \alpha^{\prime}=d_{1}\left(d_{1} \alpha^{\prime}\right)=d_{1} \alpha^{*}=\alpha, N y=d_{0}^{* 2} N y^{\prime}=d_{0}^{* 2} \beta^{\prime}=\beta$, $i \bar{x} y=$ $i d_{1} d_{0}^{*} \bar{x}^{\prime} y^{\prime}=d_{1} d_{0}^{*} \gamma^{\prime}=d_{0} \gamma^{\prime}=\gamma$. Hence we still have $f_{t, Z}^{-1}(\sigma) \neq \emptyset$ in this case.

We are now ready to prove the following criterion for the existence of solutions. For $\sigma=(\alpha, \beta, \gamma) \in \Sigma(t)_{z}$, put $\Delta_{\sigma}=\left(\alpha, \beta, \gamma_{0}, \gamma_{1}\right)$ where $\gamma=\gamma_{0}+\gamma_{1} i$. Then we have

$$
f_{t, Z}^{-1}(\sigma) \neq \emptyset \Leftrightarrow \Delta_{\sigma} \in N(Z[i]) \text {. }
$$

Proof. When $\gamma=0$, we have $\Delta_{\sigma}=(\alpha, \beta)=t$ and the assertion is nothing but (3.1). Hence, from now on, we shall assume that $\gamma \neq 0$. $\Leftrightarrow$ ) Take $z=x+y j \in S^{3}(t)_{z}$ such that $f_{t}(z)=\sigma$. Thus we have $\alpha=N x$, $\beta=N y, \gamma=i \bar{x} y$. Put $\alpha=\Delta_{o} \alpha^{\prime}, \beta=\Delta_{\sigma} \beta^{\prime}, \gamma_{0}=\Delta_{\sigma} \gamma_{0}^{\prime}, \gamma_{1}=\Delta_{\sigma} \gamma_{1}^{\prime}$. Then, by the argument in Case 3, there are $x^{\prime}, y^{\prime} \in Z[i]$ such that $N x^{\prime}=\alpha^{\prime}, N y^{\prime}=\beta^{\prime}$, $\gamma^{\prime}=i \bar{x}^{\prime} y^{\prime}$, where $\gamma^{\prime}=\gamma_{0}^{\prime}+\gamma_{1}^{\prime} i$. Since $\alpha=\Delta_{o} \alpha^{\prime}$, we have $N x=\Delta_{o} N x^{\prime}$, i.e. $\Delta_{\sigma}=N\left(x / x^{\prime}\right)$. Then we have $\Delta_{\sigma}=N \delta, \delta \in Z[i]$, e.g. by the lemma of Davenport-Cassels applied to the binary form $X^{2}+Y^{2}{ }^{*}$ )

$(\Leftrightarrow)$ Let $x^{\prime}, y^{\prime}$ be as in the proof of $(\Rightarrow)$. By the assumption, there is a number $\delta \in Z[i]$ such that $\Delta_{\sigma}=N \delta$. Put $x=\delta x^{\prime}, y=\delta y^{\prime}$. Then, $N x$ $=\Delta_{\sigma} N x^{\prime}=\Delta_{\sigma} \alpha^{\prime}=\alpha, N y=\Delta_{\sigma} N y^{\prime}=\Delta_{\sigma} \beta^{\prime}=\beta, i \bar{x} y=i \bar{\delta} \bar{x}^{\prime} \delta y^{\prime}=\Delta_{\sigma} \gamma^{\prime}=\gamma$. Hence, we have $f_{t, z}(z)=\sigma$ with $z=x+y j$, q.e.d.

Translating (3.3) in terms of $h_{t, z}$, we obtain the following criterion.

*) See, e. g. J-P. Serre, Cours d’arithmétique, Paris, 1970, p. 80. 
Notation being as in $\S 2$, for $w=u i+v j \in S^{2}\left(t^{2}\right)_{Z}, u \in Z, v=v_{0}+v_{1} i$ $\in Z[i]$, we have

$$
h_{t, Z}^{-1}(w) \neq \emptyset \Leftrightarrow 2 \mid v \quad \text { and } \quad \Delta_{w} \in N(Z[i]),
$$

where $\Delta_{w}=\left(\frac{1}{2}(t+u), \frac{1}{2}(t-u), \frac{1}{2} v_{0}, \frac{1}{2} v_{1}\right)$.

\section{§4. Number of solutions}

For a finite set $F$, we denote by Card $F$ the number of elements in it. Thus $r(n)=\operatorname{Card}\left\{(x, y) \in Z^{2}, x^{2}+y^{2}=n\right\}$. Using notations in $\S 2, \S 3$, one restates the proposition (1.1) as

$$
\operatorname{Card}\left(h_{t, \boldsymbol{Z}}^{-1}(w)\right)=r\left(\Delta_{w}\right) \quad \text { for any } w \in S^{2}\left(t^{2}\right)_{\mathbf{Z}}^{\text {even }} .
$$

Translating (4.1) in terms of $f_{t, Z}$, we are reduced to prove that

$$
\operatorname{Card}\left(f_{t, \mathbf{Z}}^{-1}(\sigma)\right)=r\left(\Delta_{\sigma}\right) \quad \text { for any } \sigma \in \Sigma(t)_{\mathbf{Z}} \text {. }
$$

Proof. Put, as before, $\sigma=(\alpha, \beta, \gamma)$. In case $\gamma=0$, since $\Delta_{\sigma}=t$, (4.2) follows from the argument in $\S 3$, Case 1. Hence, from now on, we shall assume that $\gamma \neq 0$. Since we already have the criterion (3.3), it is enough to consider the case where $f_{t, Z}^{-1}(\sigma) \neq \emptyset$. So, take a point $z=x+y j \in f_{t, Z}^{-1}(\sigma)$ and call $I_{z}$ the ideal in $Z[i]$ generated by $x$ and $y: I_{z}=Z[i] x+Z[i] y$. Let $z^{\prime}=x^{\prime}+y^{\prime} j$ be another point in the same fibre as $z$. We want to compare $I_{z}$ and $I_{z^{\prime}}$. Since $f_{t, Z}(z)=f_{t, z}\left(z^{\prime}\right)$, we have $N x=N x^{\prime}, N y=N y^{\prime}, \bar{x} y=\bar{x}^{\prime} y^{\prime}$. From these relations, we see that there is an element $\rho \in \boldsymbol{Q}(i)$ with $N \rho=1$ such that $x^{\prime}=\rho x, y^{\prime}=\rho y$. It then follows that $I_{z^{\prime}}=\rho I_{z}$ and so $N I_{z^{\prime}}=N I_{z}=n_{\sigma}$, a natural number depending only on $\sigma \in \Sigma(t)_{z}$. For a natural number $n$, Put:

$$
\Theta(n)=\{\theta \in Z[i], N \theta=n\} .
$$

Hence we have $\operatorname{Card}\left(\Theta\left(n_{\sigma}\right)\right)=r\left(n_{\sigma}\right)$. We shall show that there is a bijection between $f_{t, \boldsymbol{Z}}^{-1}(\sigma)$ and $\Theta\left(n_{\sigma}\right)$. To do this, fix a point $\zeta=\xi+\eta j$ $\in f_{t, Z}^{-1}(\sigma)$ and, for any $z=x+y j \in f_{t, Z}^{-1}(\sigma)$, denote by $\rho_{z}$ the element in $\boldsymbol{Q}(i)$ with $N \rho_{z}=1$ such that $x=\rho_{z} \xi, y=\rho_{z} \eta$. Since $Z[i]$ is a principal ideal ring, there is an element $\omega \in Z[i]$ such that $I_{\zeta}=(\omega)$. Put

$$
T(z)=\omega \rho_{z}, \quad z \in f_{t, Z}^{-1}(\sigma) .
$$

We claim that $T$ is the bijection we are looking for. First of all, write $\omega=\lambda \xi+\mu \eta, \lambda, \mu \in \boldsymbol{Z}[i]$. Then, $T(z)=\omega \rho_{z}=\lambda \xi \rho_{z}+\mu \eta \rho_{z}=\lambda x+\mu y \in \boldsymbol{Z}[i]$ 
and $N(T(z))=N \omega=N I_{\xi}=n_{o}$, which shows that $T$ maps $f_{t, Z}^{-1}(\sigma)$ into $\Theta\left(n_{o}\right)$. Next, assume that $T(z)=T\left(z^{\prime}\right)$. Then, we have $\rho_{z}=\rho_{z^{\prime}}$ and hence $x=x^{\prime}, y=y^{\prime}$, i.e. $z=z^{\prime}$. To see that $T$ is surjective, take any $\theta \in \Theta\left(n_{\sigma}\right)$ and put $x=\theta \omega^{-1} \xi, y=\theta \omega^{-1} \eta, z=x+j y$. Since $I_{\xi}=(\omega)$, we have $\xi$ $=a \omega, \eta=b \omega$ with $a, b \in Z[i]$. It follows that $x=\theta a$ and $y=\theta b$ both belong to $Z[i]$. Now, since $N x=N(\theta) n_{\sigma}^{-1} N \xi=N \xi=\alpha, N y=N(\theta) n_{\sigma}^{-1} N \eta$ $=N \eta=\beta$, we have $N z=N x+N y=\alpha+\beta=t$, i.e. $z \in S^{3}(t)_{z}$. Furthermore, we have $i \bar{x} y=i \bar{\theta} \bar{\omega} \bar{\omega}^{-1} \bar{\xi} \theta \omega^{-1}=i N(\theta) n_{\sigma}^{-1} \bar{\xi} \eta=i \bar{\xi} \eta=\gamma$, which shows that $z \in f_{t, Z}^{-1}(\sigma)$. Finally, since $x=\theta \omega^{-1} \xi, y=\theta \omega^{-1} \eta$, we have $\rho_{z}=\theta \omega^{-1}$ and so $T(z)=\rho_{z} \omega=\theta$, which completes the proof of the surjectivity of $T$. In order to complete the proof of (4.2), we must show that

$$
n_{\sigma}=\Delta_{\sigma} \quad \text { whenever } f_{t, \boldsymbol{Z}}^{-1}(\sigma) \neq \emptyset .
$$

First, observe that $I_{\zeta} \bar{I}_{\zeta}=\left(n_{o}\right)$ and so $n_{\sigma}=\left(\xi \bar{\xi}, \eta \bar{\eta}, \xi \bar{\eta}+\bar{\xi}_{\eta}\right)=\left(\alpha, \beta, 2 \gamma_{1}\right)$. From the relation $\alpha \beta=\gamma_{0}^{2}+\gamma_{1}^{2}$, one sees easily that $n_{\sigma}$ and $\Delta_{\sigma}$ contain each odd prime $p$ with the same exponent. Hence, it remains to examine the exponent of 2 . Denote by $\nu_{2}(a)$ the exponent of 2 in an integer $a$. Since we obviously have $\nu_{2}\left(\Delta_{\sigma}\right) \leqq \nu_{2}\left(n_{\sigma}\right)$, it is enough to show that $\nu_{2}\left(n_{\sigma}\right)$ $\leqq \nu_{2}\left(\Delta_{\sigma}\right)$. Hence, we may assume that $\nu_{2}\left(n_{\sigma}\right) \geqq 1$. Put $e=\nu_{2}\left(n_{\sigma}\right)$ and write $\alpha=2^{e} \alpha^{*}, \beta=2^{e} \beta^{*}, \gamma_{1}=2^{e-1} \gamma_{1}^{*}$ and $\gamma_{0}=2^{f} \gamma_{0}^{*}$ with $\left(2, \gamma_{0}^{*}\right)=1$. We have then $2^{2 e} \alpha^{*} \beta^{*}=2^{2 f} \gamma_{0}^{* 2}+2^{2(e-1)} \gamma_{1}^{* 2}$, or $2^{2 f} \gamma_{0}^{* 2}=2^{2(e-1)}\left(4 \alpha^{*} \beta^{*}-\gamma_{1}^{* 2}\right)$. If $\gamma_{1}^{*}$ were odd, we must have $f=e-1$, and then $4 \alpha^{*} \beta^{*}=\gamma_{0}^{* 2}+\gamma_{1}^{* 2}$, which is impossible because both of $\gamma_{0}^{*}, \gamma_{1}^{*}$ are odd. Therefore, $\gamma_{1}^{*}$ must be even and so we have $e \leqq \inf \left(\nu_{2}\left(\gamma_{1}\right), \nu_{2}\left(\gamma_{0}\right)\right)$, which implies that $\nu_{2}\left(n_{\sigma}\right)$ $\leqq \nu_{2}\left(A_{\sigma}\right)$, q.e.d. 